\title{
A satisfactory quantitative test of lymphocyte response to phytohaemagglutinin for the definition of normal control values and recognition of immunological defects
}

\author{
MARGARET G. FITZGERALD \\ From the Pathology Department, Royal Children's Hospital, Victoria, Australia
}

SYNOPSIS Despite widespread doubts about the quantitative validity or clinical usefulness of lymphocyte response to phytohaemagglutinin (PHA), a satisfactory quantitative test of such responsiveness suitable for the clinical recognition of immunological defects has been developed here. This was achieved by exploring and controlling technical and other variables in the culture of lymphocytes and the quantitation of their response to phytohaemagglutinin. The aspects evaluated included intraperson as well as person-to-person variations, non-lymphocytic cell content, lymphocyte number, PHA batch, atmospheric conditions, culture duration, and quantitation of response. As a result of the information gained from these studies, together with the normal dose-response curve previously established (Fitzgerald, 1971), a satisfactory quantitative and reproducible method suitable for routine clinical use has been realized. This has been applied to the investigation of patients with suspected immunological deficiency disorders, and significant deviations from the normal have been shown. In addition, a PHA dose-response ratio derived from the responses of normal individuals and patients gives a practical quantitative expression of such defects.

As yet there is no satisfactory quantitative method for clinical assessment of cell-mediated immunity in patients with suspected immunological deficiency disorders. The response of lymphocytes to PHA in culture appears to correlate closely with other indices of cellular immune competence (Lischner, Punnett, and DiGeorge, 1967), although the precise mechanism of the reaction is still far from understood. Various workers have raised doubts about the value of the method as a measure of lymphocyte reactivity of use in clinical assessment, possibly contributed to by variations in technique between laboratories (Naspitz and Richter, 1968; Pentycross, 1969; Oppenheim, Blaese, and Waldmann, 1970). These doubts have been further augmented by the inadequancy both of control data and of comparative analysis between normal and abnormal lymphocyte responses.

The ultimate aim of this method is to provide the most sensitive discrimination between immunologicReceived for publication 5 July 1971. ally normal individuals and patients with immune defects, while at the same time keeping the test within practical bounds as a routine laboratory procedure rather than a specialized research investigation. The studies presented here have explored possible sources of variability in the response and shown that optimal practical conditions for these can be satisfactorily defined and controlled. As a result, a satisfactory method of quantitation allowing a high degree of reproducibility and readily applicable to clinical investigation has been achieved.

The technical aspects of lymphocyte culture evaluated in this study include intraperson as well as person-to-person variations, cellular content of the cultures, PHA batch, atmospheric conditions, culture duration, and quantitation of response. Examples of the application of this lymphoctye culture method to a variety of clinical immunological deficiencies are included. A more detailed description of these abnormal responses is presented elsewhere (Hosking, Fitzgerald, and Simons, 1971). 


\section{Materials and Methods}

Our routine method for culture of lymphocytes and quantitation of their response to the mitogen phytohaemagglutinin (PHA) has been detailed elsewhere (Fitzgerald, 1971). In brief, one million lymphocytes in leucocyte suspension were cultured in $1 \mathrm{ml}$ medium for 72 hours. A range of PHA (Wellcome brand) concentrations was used calculated on $50 \mathrm{mg}$ protein/5 ml (Marshall and Norins, 1965). Quantitation of the response was made using a DNA precursor.

Intraperson variation and technical reproducibility were investigated, interperson variation having been determined earlier (Fitzgerald, 1971).

Experiments to ascertain the effect of a decreased non-lymphocytic concentration in the culture have been previously reported (Fitzgerald, 1971). Parallel culture series were set up to test the influence on the response of the number of lymphocytes present in the culture.

Other culture variables investigated were time delay before setting up the cultures, PHA batch, atmosphere in the culture tubes, and culture duration. Serum in the medium was considered briefly.

Exposure time of cells to tritiated thymidine $\left({ }^{3} \mathrm{H}-\mathrm{T}\right)$ and its concentration in the medium were aspects of the quantitation technique studied.

A quench correction curve was constructed relating automatic external standard to percentage counting efficiency. Its basis was the routine culture procedure with the addition of varying amounts of red cells, because colour provided the major quench source in this system. Quenching by hyamine (Radin, 1958) was regarded as a constant factor.

Removal of red cells before culture (Bach and Voynow, 1966) or during the harvesting procedure (Junge, Hoekstra, Wolfe, and Deinhardt, 1970) both proved effective in eliminating effects due to colour.

\section{Results and Discussion}

VARIATION AND REPRODUCIBILITY Variation due to the lymphocytes has been noted in the form of interperson and intraperson variation. Richter and Naspitz (1967) showed a varying response by lymphocytes of different individuals to a range of PHA concentrations. Pentycross (1969) gave a normal range of $36-60 \%$ lymphocyte transformation with one PHA dose and Gotoff (1968) a range of $160-848 \mathrm{dpm} \times 10^{3} / 3 \times 10^{6}$ mononuclear cells. Oppenheim et al (1970) plotted mean and standard error over a PHA concentration range. Our dose-response curve, giving geometric mean and standard deviation for 25 normal adults at seven

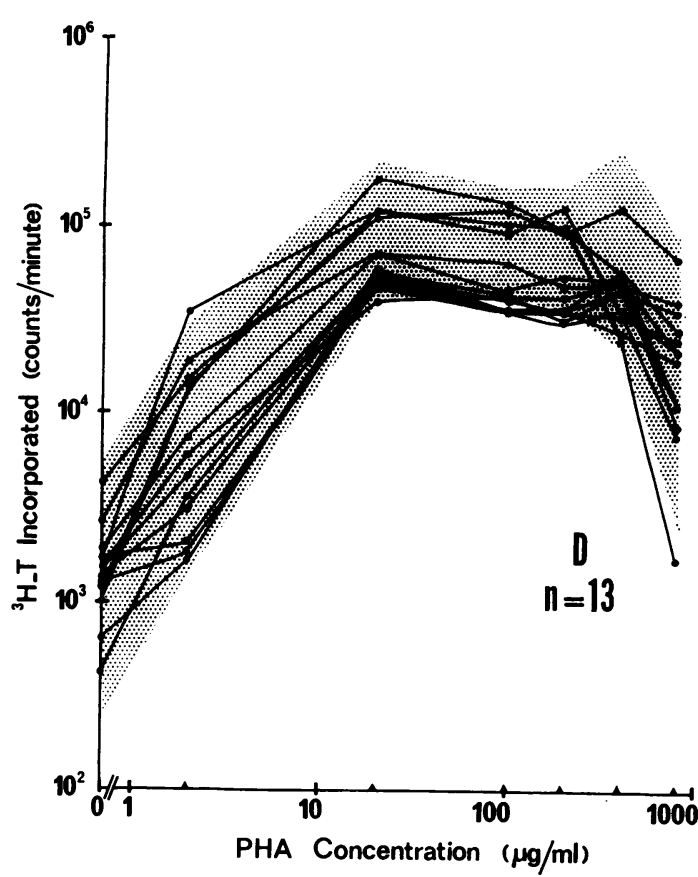

Fig. 1 Response obtained in 13 cultures of person D over a 12-month period (- mean of duplicate culture Shaded area: normal population dose-response curve $\pm 2 S D$.

PHA concentrations, has been reported (Fitzgerald, 1971).

Repeated cultures using the lymphocytes of one individual also showed variation and the extent was measured by Junge et al (1970) They used whole blood micropreparations and reported a variation of 211 to 297 times control for one person's cells cultured four times at four weekly intervals. McIntyre and Cole (1969) found a range of 20 to $160 \%$ ${ }^{32} \mathrm{P}$ uptake $\times 10^{-3}$ at one PHA dose for one individual seven times over six weeks. However, statistical analysis of the variation is possible and has been made for the dose-response curves of four individuals prepared over a 12-month period. All the curves fell within the interperson standard curve area (curves of D Fig. 1), although the geometric standard deviation was slightly wider.

Response according to the number of lymphocytes present in culture has been found to vary by such 2 workers as Schellekens and Eijsvoogel (1968), McIntyre and Cole (1969), and Siagian (1969). Their results accorded with those of Watkins and Moorhead (1969) who related the degree of response to cell crowding and surface area. They found an increase from $2 \times 10^{5}$ to $1 \times 10^{8}$ lymphocytes on a 


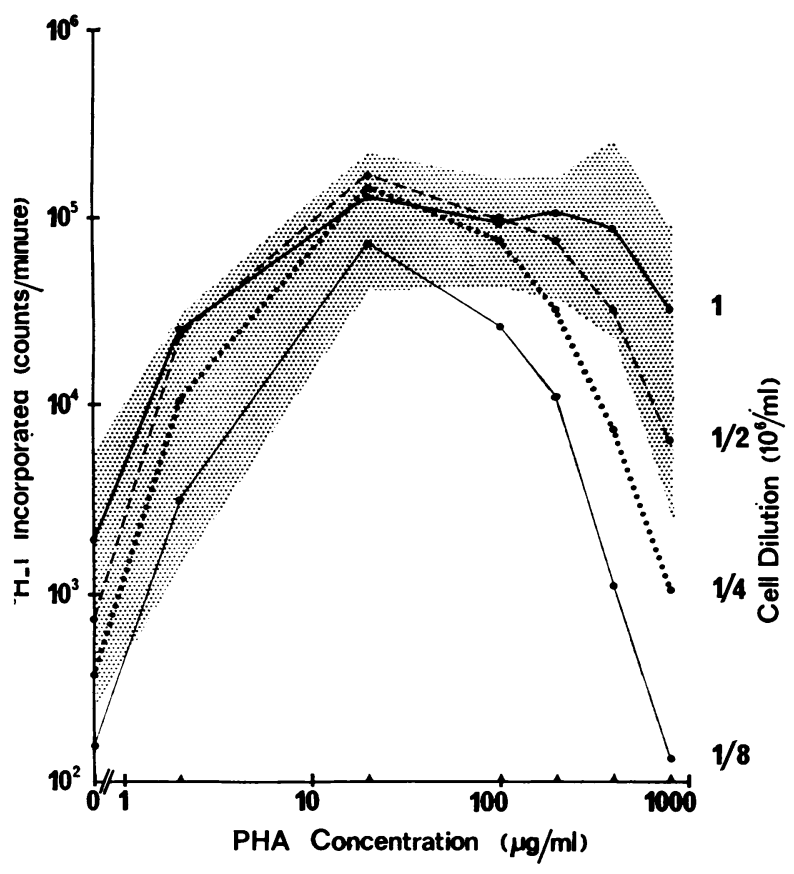

Fig. 2 Absolute counts of a representative series of cultures with differing cell numbers (cell dilution 1 represents $10^{6} \mathrm{cells} / \mathrm{ml}$, routine standard) (shaded area as for Figure 1).

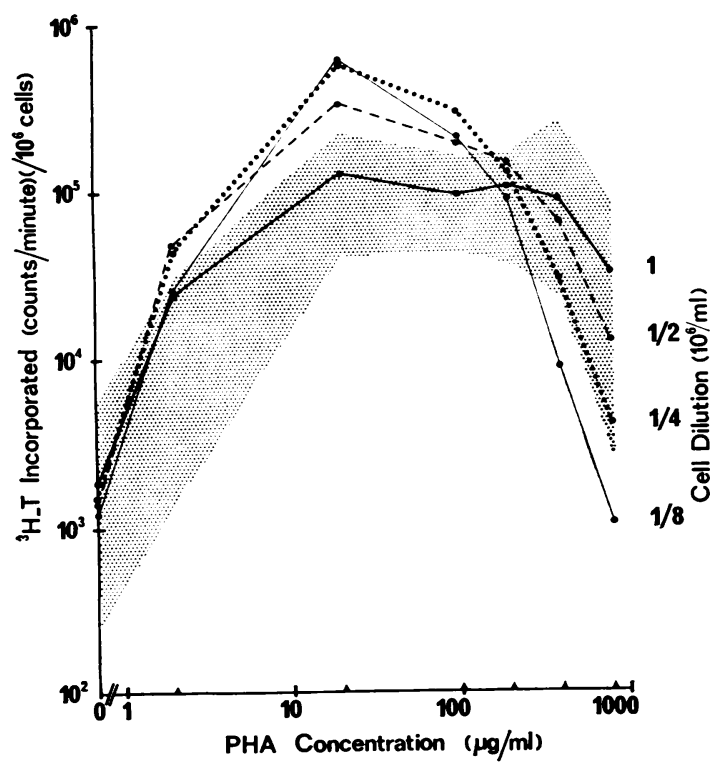

Fig. 3 Derived counts of the same representative series as in Fig. 2 (response calculated per million cells for each dilution) (shaded area as for Figure 1). surface area of 50 to $100 \mathrm{~mm}^{2}$ increased the response 15 to 260 times. Further increases resulted in a relative decrease in activity. Our results plotted as absolute counts (Fig. 2), or as derived counts with response calculated per million cells for each cell dilution (Fig. 3), similarly showed that the response was equivalent at the four lymphocyte dilutions. In fact, the concentration of $1 \times 10^{6}$ cells employed by many workers results in submaximal response, although its use for routine purposes is still valid. Maximal response, however, is obtained with $\frac{1}{2} \times 10^{6}$ and this cell concentration may be preferable for finer discrimination of response as discussed later.

\section{CULTURE CONDITIONS}

It was found that if a time delay between collection of blood and the start of the stimulation of the cells is unavoidable, then storage at room temperature with a delay of not more than $24 \mathrm{hr}$ provides the best conditions for subsequently separating cells and obtaining growth. Brown (1971) has evaluated current methods of storing human blood before whole blood culture and concluded that DNA synthesis was near maximum for samples set up immediately. The 24-hr storage condition generally best suited for culture was storage in medium; holding blood at room temperature or at $6^{\circ} \mathrm{C}$ tended to be poorer.

The responses obtained with different batches of PHA all fell within 2 standard deviations of the response of the batch routinely used (Fig. 4). No major differences appeared to result from the use of different batches of Burroughs Wellcome lot $\mathrm{K}$ phytohaemagglutinin.

More efficient conditions prevailed (Fig. 5) when the atmosphere in which the cells were cultured was $5 \% \mathrm{CO}_{2} / 95 \%$ air than when the tubes were stoppered. Dutton and Eady (1964) preferred $5 \% \mathrm{CO}_{2}$ in oxygen for studying antigenic stimulation. Wilson and Thomson (1968) found that $\mathrm{CO}_{2}$ in the air phase of cultures was needed to prevent death of leucocytes.

Without any serum in the medium, deficient growth at all PHA concentrations was evident. The batch of the calf serum was not systematically investigated, but the three batches used have not resulted in any apparent difference. Schellekens and Eijsvoogel (1968) found that $20 \%$ serum gave optimal results. They showed also that there were no significant differences between serum sources after three-day PHA stimulation, although foetal calf serum resulted in some stimulation in six-day nonPHA cultures. Ling (1968) and Junge et al (1970) recorded higher responses when autologous rather than foetal calf serum was used.

The maximum DNA synthetic period lies between 54 and $72 \mathrm{hr}$ (Richter and Naspitz, 1967; Schellekens 

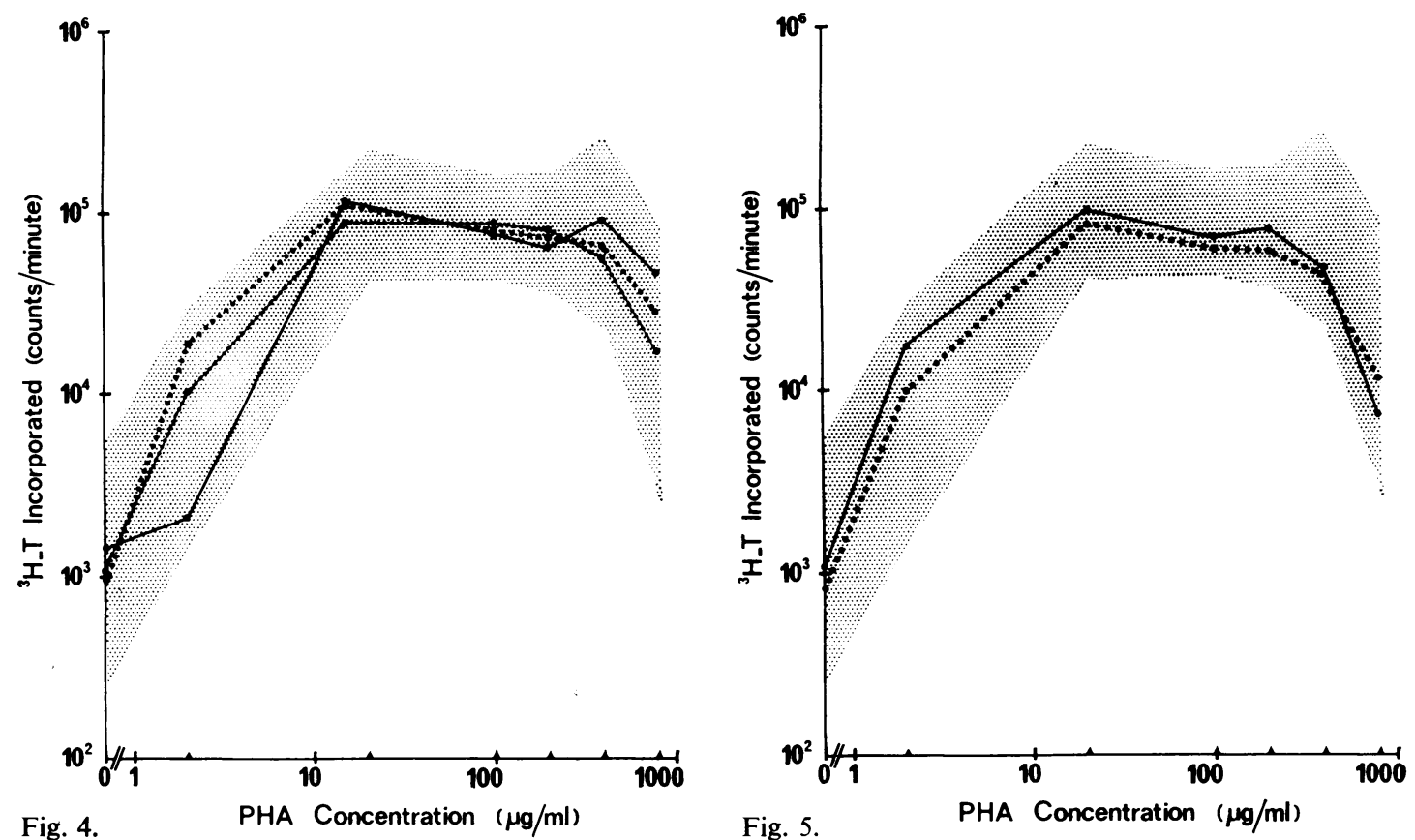

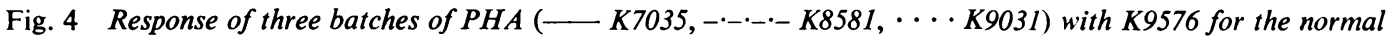
dose-response curve, shaded area. Points represent mean of three separate repetitions in duplicate.

Fig. 5 Atmospheric condition in culture tube (- $-5 \% \mathrm{CO}_{2} / 95 \%$ air, $\cdots$ stoppered tube). Points represent mean of four (shaded area as for Figure 1).

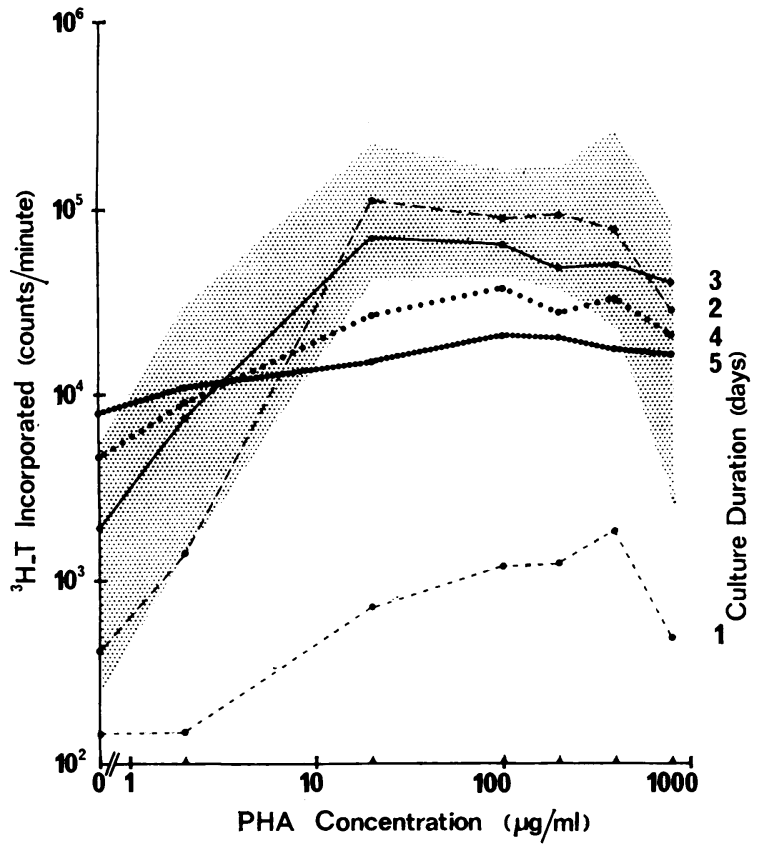

Fig. 6 Representative result for the response of lymphocytes in culture for one to five days (shaded area as for Figure 1). and Eijsvoogel, 1968; Siagian, 1969; Rubin, 1970; Brown, 1971), although in the system used by McIntyre and Cole (1969) the peak response appeared to occur at seven days. Our comparative series of culture durations consistently showed a maximum response at $48 \mathrm{hr}$ (Fig. 6).

RADIOACTIVE QUANTITATION

Incorporation of thymidine-2-14 $\mathrm{C}$ (specific activity $12.5 \mathrm{mCi} / \mathrm{mM}$ ) during the $24-\mathrm{hr}$ period 48 to 723. hours) culture was shown to be linear by Schellekens $\delta$ and Eijsvoogel (1968). The investigation of thymidine concentration revealed a dose effect below 0 $2.9 \mu \mathrm{g} / \mathrm{ml}$. Bain (1970) observed that ${ }^{3} \mathrm{H}-\mathrm{T}$ concentration, and the time for which the cells are exposed, were aspects of quantitation of the response that $\bar{N}$ could be varied and still give adequate results. This $\odot$ coincides with our results (Figs. 7 and 8).

In ascertaining the reproducibility of the technique, Schellekens and Eijsvoogel (1968) calculated the $O$ coefficient of variation for 450 cultures prepared in triplicate and found that $84 \%$ had coefficients of variation of $15 \%$. The geometric mean and standard $\stackrel{\odot}{\rightarrow}$ deviation from our experiment with 10 replicates per $O$ PHA concentration (Fig. 9) indicates that good ${ }_{0}^{\circ}$ technical reproducibility is attainable and it is $\overparen{\mathbb{D}}$ adequate to average the results of duplicate cultures. 


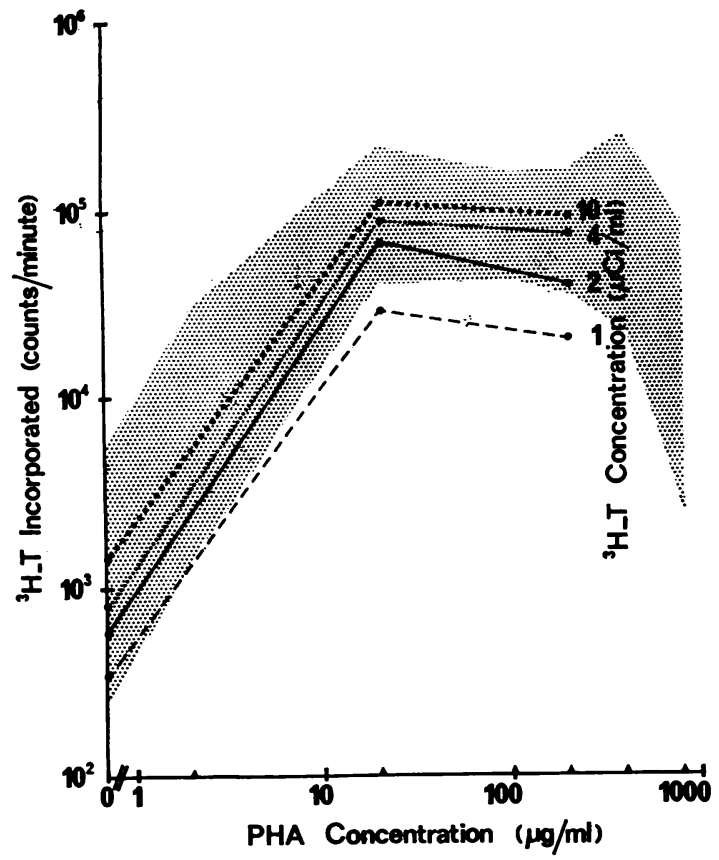

Fig. 7 Response with increasing concentrations (1-10 $\mu \mathrm{Ci}$ ) of ${ }^{3} \mathrm{H}-\mathrm{T}$ as growth indicator (shaded area as for Figure 1).

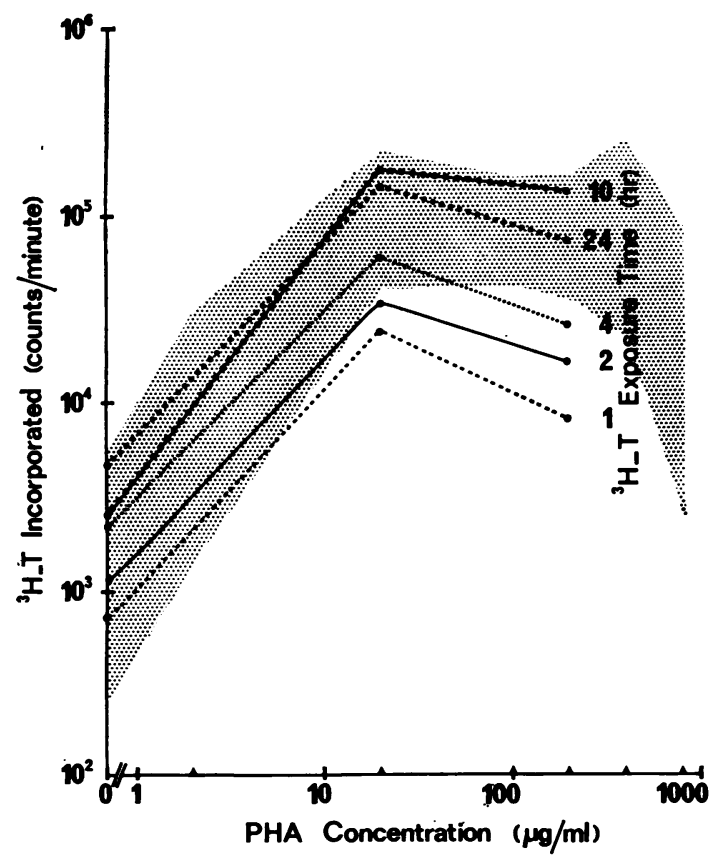

Fig. 8 Response with increasing exposure time (1-24 hr) of the cells to ${ }^{3} \mathrm{H}-\mathrm{T}$ (shaded area as for Figure 1 ).
TYPE AND NUMBER OF LEUCOCYTES

The effect that cells other than lymphocytes exert in the response has shown to be significant by Levis and Robbins (1970) when a markedly reduced response by purified lymphocytes to antigens and PHA was restored by the addition of glass-adherent cells. In contrast, Schellekens and Eijsvoogel (1968) indicated that the addition of granulocytes to purified PHAstimulated lymphocytes does not significantly alter the ${ }^{14} \mathrm{C}$-thymidine uptake and we have demonstrated

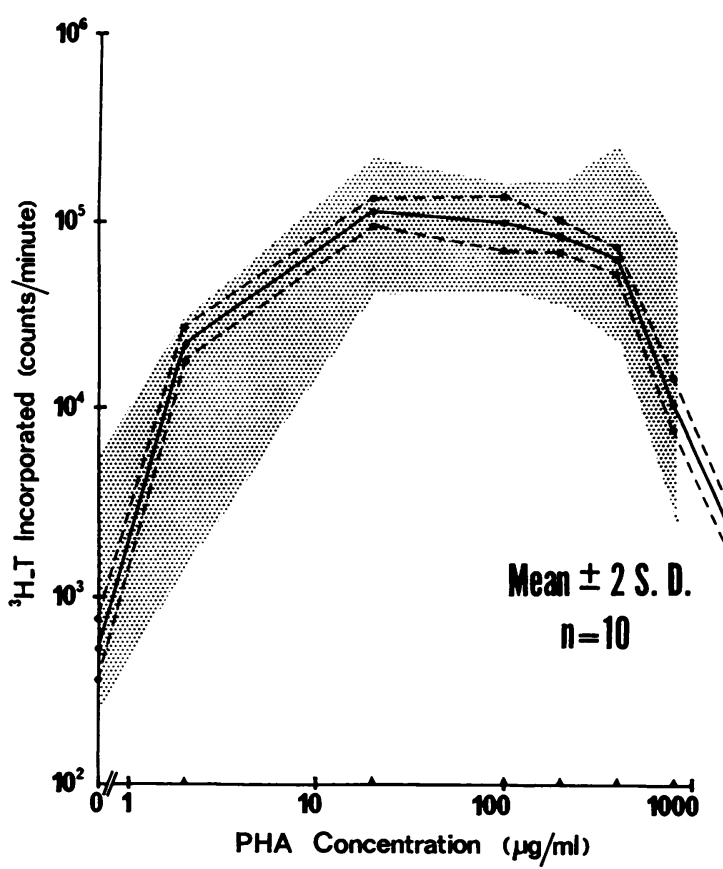

Fig. 9 Dose-response curve of one individual, 10 replicates at each PHA concentration (- mean, --$\pm 2 S D$ ) (shaded area as for Figure 1).

that elaborate purification of the leucocyte preparation is unnecessary (Fitzgerald, 1971). The intraperson culture series further enforces this, for no correlation was found between the level of response and the non-lymphocytic content $(30-70 \%)$ of the preparation. McIntyre and Cole (1969) reached a similar conclusion in reference to their interperson variation.

\section{CLINICAL APPLICATION}

The object of these technical studies was to develop the simplest test on the smallest volume of blood which would provide the most sensitive differentiation between immunologically normal individuals and patients with disorders of immune mechanisms. There appear to be two factors of importance in this 
differentiation and they frequently seem to parallel each other. They are the height of the curve compared with normal and the shape of the curve over the 'plateau' region (200-400 $\mu \mathrm{g}$ PHA/ml medium). The latter has been given mathematical expression as the PHA dose-response ratio, ie,

$\log$ response at $200 \mu \mathrm{g}$ PHA minus control count

$\log$ response at $20 \mu \mathrm{g}$ PHA minus control count

It provides another means for analysing normal variation (Fig. 10), assessing technical aspects of the response, and determining patient response.

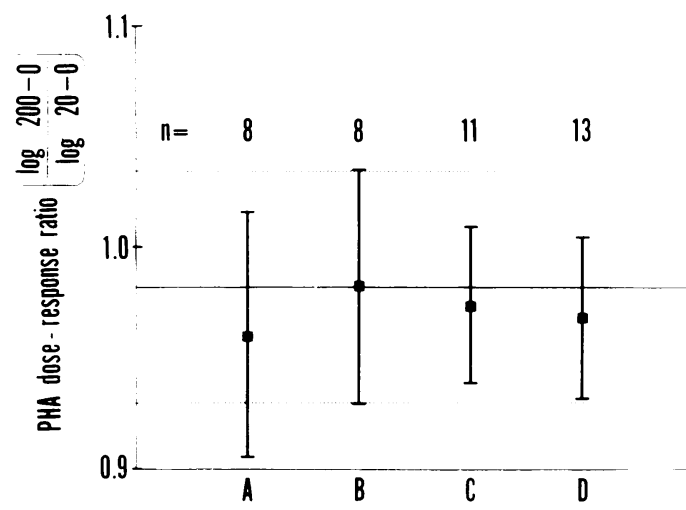

Fig. 10 Normal adult PHA dose-response ratio (- mean, $\cdots \pm 2 S D$ ) with intraperson doseresponse ratio of individuals $A, B, C$, and $D$ replicated $n$ times $(|--\square-|$ mean $\doteqdot 2 S D)$.

From these considerations, it appears that the practical system outlined here, although not invalidated in any way, could be even further simplified and modified in several directions. These include the use of $\frac{1}{2} \times 10^{6}$ instead of $1 \times 10^{6}$ lymphocytes per culture, and a culture duration of two rather than three days. They may incorporate a minimum PHA range with concentrations 0,20 , and $200 \mu \mathrm{g}$, because seven concentrations appear unnecessary at present. The future may show that more information will be obtainable from more points in the above or a wider PHA concentration range. Results so far, however, suggest that such a range is able to supply most of the relevant information concerning height of response and dose-response ratio.

The quantitative routine method of lymphocyte culture outlined has shown its reproducibility and susceptibility to analysis. The technique has been applied up till now in its routine form to patients with suspected immunological deficiency disorders. Even in its present form the technique has demon- strated defects of lymphocyte reactivity in vitro in six patients with ataxia telangiectasia, and in seven patients with infantile sex-linked agammaglobulinaemia (Hosking et al, 1971). The evaluation of the sources of variability in the response has led to a more reliable interpretation of the results of patients' lymphocyte responses in culture. As well it has achieved a more satisfactory technique for clinical investigation which allows sensitive discrimination between immunologically normal individuals and patients with immunological deficiency disorders.

References

Bach, F. H., and Voynow, N. K. (1966). One-way stimulation in mixed leukocyte cultures. Science, 153, 545-547.

Bain, B. (1970). Tritiated-thymidine uptake in mixed leucocyte $N$ cultures: effect of specific activity and exposure time. Clin. exp. Immunol., 6, 255-262.

Brown, J. K. (1971). Personal communication.

Dutton, R. W., and Eady, J. D. (1964). An in vitro system for the study of the mechanism of antigenic stimulation in the secondary response. Immunology, 7, 40-53.

Fitzgerald, M. G. (1971). The establishment of a normal human population dose-response curve for lymphocytes cultured with PHA (Phytohaemagglutinin). Clin. exp. Immunol., 8, 421-425

Gotoff, S. P. (1968). Lymphocytes in congenital immunological deficiency diseases. Clin. exp. Immunol. 3, 843-856.

Hosking, C. S., Fitzgerald, M. G., and Simons, M. J. (1971). Clin. exp. Immunol., 9, 467-476.

Junge, U., Hoekstra, J., Wolfe, L., and Deinhardt, F. (1970). Microtechnique for quantitative evaluation of in vitro lymphocyg transformation. Clin. exp. Immunol., 7, 431-437.

Levis, W. R., and Robbins, J. H. (1970). Effect of glass-adherent ce on the blastogenic response of 'purified' lymphocytes phytohaemagglutinin. Exp. Cell. Res., 61, 153-158.

Ling, N. R (1968), Lymphocyte Stimulation. North-Holland Publishing Company, Amsterdam.

Lischner, H. W., Punnett, H. H., and DiGeorge, A. M. (1967). Lymphocytes in congenital absence of the thymus. Nature (Lond.), 214, 580-582.

McIntyre, O. R., and Cole, A. F. (1969). Variation in the response of normal lymphocytes to PHA. Int. Arch. Allergy, 35, 105-118.

Marshall, W. H., and Norins, L. C. (1965). Antigenic properties of the extract of Phaseolus vulgaris seeds (phytohaemagglutinin) routinely used in leucocyte cultures. Aust. J. exp. Biol, med. Sci., 43, 213-228.

Naspitz, C. K., and Richter, M. (1968). The action of phytohaemagglutinin in vivo and in vitro: a review. Progr. Allergy, 12, 1-85.

Oppenheim, J. J., Blaese, R. M., and Waldmann, T. A. (1970). Defective lymphocyte transformation and delayed hypersensitivity in Wiskcott-Aldrich syndrome. $J$. Immunol., 104, 835-844.

Pentycross, C. R. (1969). Lymphocyte transformation in young people. Clin. exp. Immunol., 5, 213-216.

Radin, N. S. (1958). In Liquid Scintillation Counting, edited by C. G. Bell and F. N. Hayes, p. 108. Pergamon Press, Oxford and New York.

Richter, M., and Naspitz, C. K. (1967). The variation in response of human peripheral lymphocytes to phytohaemagglutinin in vitro. Int. Arch. Allergy, 32, 288-293.

Rubin, A. D. (1970). Ribosome biosynthesis in cultured lymphocytes: II. The role of ribosomal RNA production in the initiation and maintenance of lymphocyte growth. Blood, 35, 708-720.

Schellekens, P. T. A., and Eijsvoogel, V. P. (1968). Lymphocyte transformation in vitro. 1 . Tissue culture conditions and quantitative measurements. Clin. exp. Immunol., 3, 571-584.

Siagian, E. G. (1969). DNA Synthesis in Human Peripheral Blood Lymphocyte Cultures. Australian Atomic Energy Commission Report, TM506.

Watkins, S. M., and Moorhead. J. F. (1969). The effect of cell crowding on the in vitro reactivity of normal and abnormal human lymph ocytes. Cell Tissue Kinet., 2, 213-223.

Wilson, J. D., and Thomson. A. E. R. (1968). Death and division of lymphocytes. Lancet, 2, 1120-1123. 